\title{
ANTAGONISTIC PLEIOTROPY, DOMINANCE, AND GENETIC VARIATION*
}

\author{
MICHAEL R. ROSE \\ Biology Department, Dalhousie University, Halifax, Nova Scotia, \\ Canada B3H $4 J 1$
}

Received 28.iv.81

\begin{abstract}
SUMMARY
Although the importance of antagonistic pleiotropy of genes affecting components of fitness has long been argued, explicit population genetic models involving pleiotropy have only recently been developed. Simple, deterministic models of this kind are given, and then analyzed for conditions sufficient for protected polymorphism. It is found that dominance in gene action plays a key role in fostering the establishment of polymorphism in these models. However, the genetic variance for individual fitness components generally remains predominantly additive in spite of this. Though these models are not very robust, they do illustrate in principle one means of explaining recent experimental findings concerning the quantitative genetics of components of fitness in populations free of inbreeding.
\end{abstract}

\section{INTRODUCTION}

THE importance of antagonistic pleiotropy in maintaining polymorphism has been pointed out many times (e.g., Caspari, 1950; Wallace, 1959; Wallace, 1968 , p. 214 ; Wallace, 1970 , p. 50; Wright, 1977, p. 557). But explicit models of the evolution of characters subject to pleiotropy have been largely lacking, excepting Charlesworth (1980) and Lande (1980). Nonetheless, for most characters this lack of theoretical attention is appropriate, since their role in determining organismal fitness is usually obscure, and their interaction with other characters equally so. Wright's classic studies of guinea pig coat colour (reviewed in Wright, 1968) constitute one of the most complete examinations of pleiotropic gene action above the molecular level, but even in this case the fitness effects of the alleles are largely unclear.

However, there is a group of characters for which the role of pleiotropy is both detectable and important: components of fitness. Characters such as female fecundity, male mating success, and zygote-to-adult viability are generally related to fitness itself in a fashion which is not as impenetrable as that of other much-studied characters, like guinea pig coat colour or Drosophila bristle number. In addition, the pattern of pleiotropy among alleles affecting such characters is evidently of great importance in the interpretation of the observed genetic variation for single fitnesscomponents. When it can be assumed that genetic effects on fecundity and similar characters are always positively correlated with effects on viability, as Mukai (1977) argues, then the partitioning of genetic components of variation for viability may be used as a guide to the genetic basis of variation in fitness itself. But if there is antagonistic pleiotropy between fitness-

* University of Wisconsin-Madison, Laboratory of Genetics, Contribution Number 2501. 
components, the relationship between genetic variation in fitnesscomponents and genetic variation in fitness becomes quite different (Falconer, 1960 , pp. 328-329, pp. 335-336). In particular, it is easy to find numerical examples in which there is abundant additive genetic variation for individual fitness-components and a complete absence of additive genetic variation in fitness itself (cf. Falconer, 1977).

Since the genetics of fitness-components are more amenable to direct investigation than the genetics of fitness itself, it is important to have some basis for interpreting the effects of antagonistic pleiotropy on genetic variation in both fitness and its components. Accordingly, this article treats simple population genetic models with fitness-components which (1) are either additive or multiplicative in their contribution to fitness and (2) are subject to genes with strictly antagonistic pleiotropic effects. The genetic systems studied are single diallelic loci, single triallelic loci, and two diallelic loci.

\section{ONE DiAllelic Locus}

(i) Genetic specification

In this case, as in all others below, the population is assumed to be infinite, randomly mating, monoecious, and diploid with discrete nonoverlapping generations. Let one allele at the locus be designated $A_{1}$ and the other $A_{2}$, so that there are the usual genotypes, $A_{1} A_{1}, A_{1} A_{2}$, and $A_{2} A_{2}$, which have the following fitness $(W)$ and fitness-component $\left(W_{i}\right)$ phenotypes.

$$
\begin{array}{cccc} 
& A_{1} A_{1} & A_{1} A_{2} & A_{2} A_{2} \\
W_{1} & V+h_{1} \varepsilon & V & V-\varepsilon \\
W_{2} & f-\delta & f & f+h_{2} \delta \\
W_{1}+W_{2} & \mathrm{~W}+h_{1} \varepsilon-\delta & \mathrm{W} & \mathrm{W}+h_{2} \delta-\varepsilon \\
\mathrm{W}=V+f) & & & \\
W_{1} W_{2} & \mathrm{~W}+h_{1} f \varepsilon & \mathrm{W} & \mathrm{W}+h_{2} V \delta \\
(\mathrm{W}=V f) & -V \delta-h_{1} \varepsilon \delta & & -f \varepsilon-h_{2} \varepsilon \delta
\end{array}
$$

All the indicated variables are taken to be non-negative. In addition, the magnitudes of $V$ and $f$ are taken to be about 1 , with $\varepsilon, \delta \ll 1$. The assumption that the $h_{i}$ are non-negative precludes overdominance in single fitnesscomponent effects. This is done in order to ensure that the maintenance of polymorphism cannot be achieved by means of overdominant effects on one fitness-component and sufficiently small effects on the other component.

\section{(ii) Polymorphism conditions with additive components}

Let $W\left(A_{i} A_{j}\right)=W_{1}\left(A_{i} A_{j}\right)+W_{2}\left(A_{i} A_{j}\right)$, giving the net fitness values indicated in row (1c) above. In this case, the necessary and sufficient condition for asymptotically stable polymorphic equilibrium due to heterozygote advantâge is

$$
\varepsilon>h_{2} \delta \text { and } \delta>h_{1} \varepsilon \text {. }
$$


For $h_{1}=h_{2}=0$ and $\varepsilon, \delta>0$, condition (2) is always met. For $h_{1} \geqq 1$ and $h_{2} \geqq 1$, condition ( 2 ) cannot be met. For $h_{i} \in(0,1)$, condition (2) can always be met for $h_{i}$ sufficiently close to zero, providing $\varepsilon, \delta>0$. Thus, some degree of directional dominance tending toward recessive deleterious genetic action is necessary for the maintenance of polymorphism. In addition, the more nearly equal $\varepsilon$ and $\delta$ are, the less dominance is required for protected polymorphism.

(iii) Polymorphism conditions for multiplicative components

Let $W\left(A_{i} A_{j}\right)=W_{1}\left(A_{i} A_{j}\right) W_{2}\left(A_{i} A_{i}\right)$, as in (1d). Heterozygote fitness advantage requires

$$
V \delta>h_{1} \varepsilon(f-\delta) \text { and } f \varepsilon>h_{2} \delta(V-\varepsilon) .
$$

For $h_{1}=h_{2}=0$ and $\varepsilon, \delta>0$, condition (3) is always met. For $h_{1}=h_{2}=1$ and $\varepsilon, \delta>0$, condition (3) requires $\varepsilon \delta>|V \delta-f \varepsilon|$. For $\varepsilon, \delta \ll 1$, as assumed, this constraint on polymorphism with additive gene action for each fitnesscomponent in turn requires $V \delta \cong f \varepsilon$ or $V / f \cong \varepsilon / \delta$. Thus some degree of dominance in individual fitness-component effects is not essential for polymorphism in this case, though strictly additive gene action places severe constraints on the parameter values required for polymorphism.

\section{(iv) Genetic analysis}

Internal equilibrium gene frequencies for $A_{1}$, say $\hat{p}$, are readily found from the standard equation for overdominant fitness systems:

$$
\hat{p}=\frac{W_{12}-W_{22}}{2 W_{12}-W_{11}-W_{22}}, \text { with } W_{i j}=W\left(A_{i} A_{j}\right) .
$$

In the additive-components case,

$$
\hat{p}=\frac{\varepsilon-h_{2} \delta}{\varepsilon\left(1-h_{1}\right)+\delta\left(1-h_{2}\right)},
$$

and for multiplicative components,

$$
\hat{p}=\frac{f \varepsilon-h_{2} \delta(V-\varepsilon)}{f \varepsilon\left(1-h_{1}\right)+V \delta\left(1-h_{2}\right)+\varepsilon \delta\left(h_{1}+h_{2}\right)}
$$

Following Falconer (1960, pp. 135-136) or Fisher (1918), the additive and dominance genetic variances for the two fitness components are obtained easily. Letting

$$
\begin{gathered}
W_{1}\left(A_{1} A_{1}\right)=a=\frac{\varepsilon\left(h_{1}+1\right)}{2}, \quad W_{1}\left(A_{1} A_{2}\right)=d=\frac{\varepsilon\left(1-h_{1}\right)}{2}, \\
\begin{array}{c}
W_{1}\left(A_{2} A_{2}\right)=-a, \quad \text { and } q=1-p, \\
V_{A}=2 p q[a+d(q-p)]^{2} \\
=2 p q \varepsilon^{2}\left(h_{1} p+q\right)^{2}
\end{array}
\end{gathered}
$$


and

$$
\begin{aligned}
V_{D} & =4 p^{2} q^{2} d^{2} \\
& =p^{2} q^{2} \varepsilon^{2}\left(1-h_{1}\right)^{2},
\end{aligned}
$$

for fitness component $W_{1}$. Likewise, for $W_{2}$,

and

$$
V_{A}=2 p q \delta^{2}\left(p+h_{2} q\right)^{2}
$$

$$
V_{D}=p^{2} q^{2} \delta^{2}\left(1-h_{2}\right)^{2} \text {, }
$$

with the same form as equations (4) and (5). Fig. 1 shows the resulting dependence of the genetic variances on the gene frequencies and the degree of dominance.

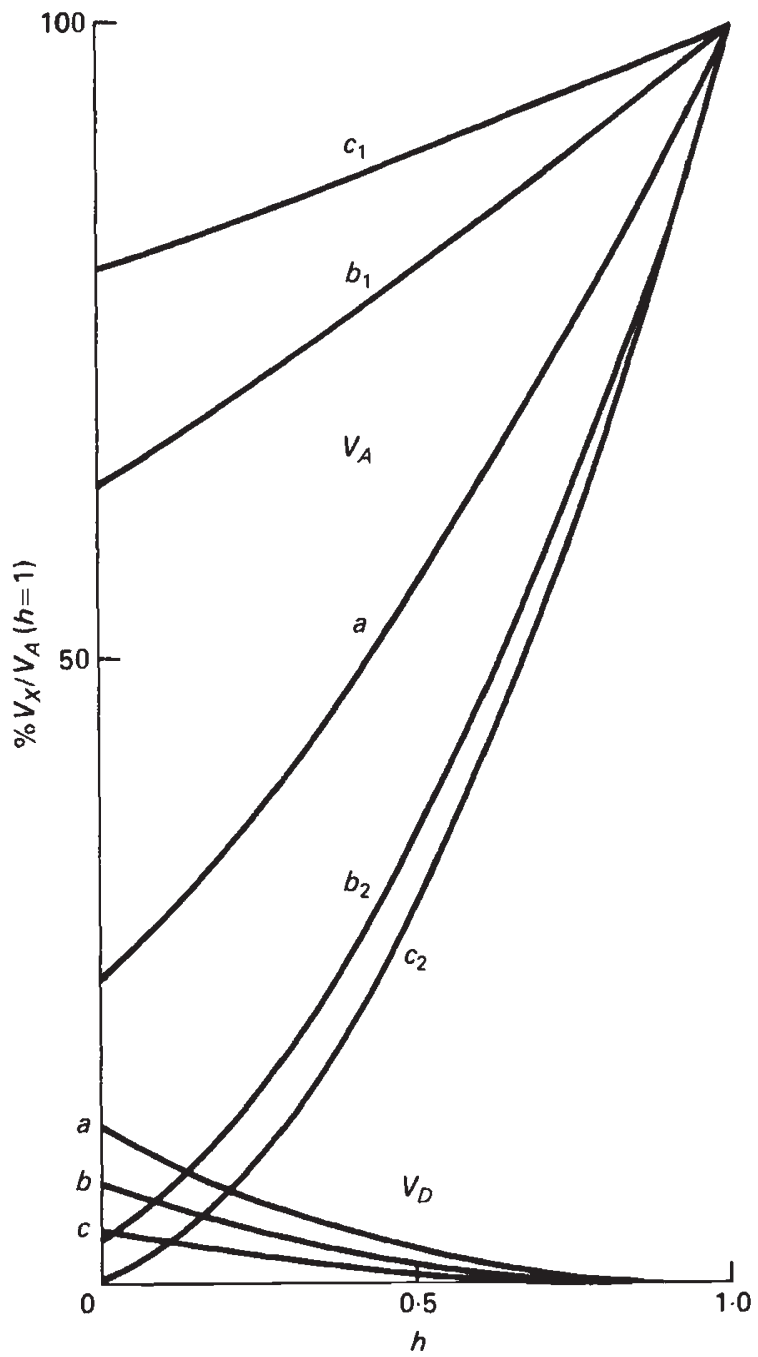

FIG. 1.-Components of genetic variance for $W_{i}$ as percentages of the additive genetic variance $\left(V_{A}\right)$ when there is no dominance in gene action, plotted as functions of the degree of dominance of gene action $(h) . V_{D}$ indicates dominance variance. For curve $a, p=q=0.5$. For curve $b_{1}, p=0.2$ and $q=0.8$, while for curve $b_{2}, p=0.8$ and $q=0.2$. For curve $b$, $p q=(0.8)(0.2)$. For $c_{1}, p=0.1$ and $q=0.9$, while for $c_{2}, p=0.9$ and $q=0.1$, and for $c$, $p q=(0 \cdot 9)(0 \cdot 1)$. 
The additive genetic correlation $\left(r_{A}\right)$ between $W_{1}$ and $W_{2}$ is also of interest, where

$$
r_{A}=\frac{\operatorname{cov}_{A}\left(W_{1}, W_{2}\right)}{\sqrt{V_{A}\left(W_{1}\right) V_{A}\left(W_{2}\right)}} \quad \text { (Falconer, 1960, p. 317). }
$$

In general,

$$
2 \operatorname{cov}(a, b)=\operatorname{var}(a+b)-\operatorname{var}(a)-\operatorname{var}(b) .
$$

For $W_{1}+W_{2}$,

$$
V_{A}=2 p q\left[\varepsilon\left(q+h_{1} p\right)-\delta\left(p+h_{2} q\right)\right]^{2} .
$$

Using equations (4), (6), (8) and (9),

$$
\operatorname{cov}_{A}\left(W_{1}, W_{2}\right)=p q\left[-2 \varepsilon \delta\left(p+h_{2} q\right)\left(q+h_{1} p\right)\right] .
$$

Since, from equations (4) and (6),

$$
\begin{aligned}
\sqrt{V_{A}\left(W_{1}\right) V_{A}\left(W_{2}\right)}= & \sqrt{2 p q \varepsilon^{2}\left(h_{1} p+q\right)^{2} 2 p q \delta^{2}\left(p+h_{2} q\right)^{2}} \\
= & 2 p q \varepsilon \delta\left(p+h_{2} q\right)\left(q+h_{1} p\right), \\
& r_{A}=-1,
\end{aligned}
$$

as might be expected from the genetic specification of model (1).

\section{ONE TRIALlELIC LOCUS}

\section{(i) Genetic specification}

Let there be one locus with three alleles, $A_{1}, A_{2}$ and $A_{3}$, with the following fitness and fitness-component phenotypes associated with the six possible genotypes. (It will be assumed that $\varepsilon_{i}>0$ and $\delta_{j}>0$, for all $i, j$.)

(a) Additive components: With $\frac{1}{2} \leqq h_{i} \leqq 1$,

$\begin{array}{cccc} & W_{1} & W_{2} & W_{1}+W_{2} \\ A_{1} A_{1} & V & f & W \\ A_{1} A_{2} & V+\varepsilon_{1} & f-\delta_{1} & W+\varepsilon_{1}-\delta_{1} \\ A_{2} A_{2} & V+2 h_{1} \varepsilon_{1} & f-2 \delta_{1} & W+2 h_{1} \varepsilon_{1}-2 \delta_{1} \\ A_{2} A_{3} & V+\varepsilon_{1}-\varepsilon_{2} & f+\delta_{2}-\delta_{1} & W+\varepsilon_{1}-\varepsilon_{2}-\delta_{1}+\delta_{2} \\ A_{3} A_{3} & V-2 \varepsilon_{2} & f+2 h_{2} \delta_{2} & W-2 \varepsilon_{2}+2 h_{2} \delta_{2} \\ A_{1} A_{3} & V-\varepsilon_{2} & f+\delta_{2} & W-\varepsilon_{2}+\delta_{2}\end{array}$

(b) Multiplicative components: With $1 \leqq h_{i} \leqq 2$,

$\begin{array}{cccc} & W_{1} & W_{2} & W_{1} W_{2} \\ A_{1} A_{1} & V & f & W \\ A_{1} A_{2} & V+\varepsilon_{1} & f-\delta_{1} & W+f \varepsilon_{1}-V \delta_{1}-\varepsilon_{1} \delta_{1} \\ A_{2} A_{2} & V+h_{1} \varepsilon_{1} & f-2 \delta_{1} & W+h_{1} f \varepsilon_{1}-2 V \delta_{1}-2 h_{1} \varepsilon_{1} \delta_{1} \\ A_{2} A_{3} & V+\varepsilon_{1}-\varepsilon_{2} & f-\delta_{1}+\delta_{2} & W+f\left(\varepsilon_{1}-\varepsilon_{2}\right)+V\left(\delta_{2}-\delta_{1}\right) \\ & & & +\left(\varepsilon_{1}-\varepsilon_{2}\right)\left(\delta_{2}-\delta_{1}\right) \\ A_{3} A_{3} & V-2 \varepsilon_{2} & f+h_{2} \delta_{2} & W-2 f \varepsilon_{2}+h_{2} V \delta_{2}-2 h_{2} \varepsilon_{2} \delta_{2} \\ A_{1} A_{3} & V-\varepsilon_{2} & f+\delta_{2} & W-f \varepsilon_{2}+V \delta_{2}-\varepsilon_{2} \delta_{2}\end{array}$


This triallelic model is not as general as the diallelic model just treated. It is based on an underlying dosage-compensation model (Wright, 1977), with alleles $A_{2}$ and $A_{3}$ having opposite effects on the net protein activity, the net effect of the two alleles together in one genotype being assumed to be small enough that each fitness component responds in a linear fashion, giving additivity for genotype $\boldsymbol{A}_{2} \boldsymbol{A}_{3}$.

\section{(ii) Polymorphism conditions for additive components}

With both genetic systems, the necessary and sufficient conditions for asymptotically stable triallelic polymorphism are:

$$
\begin{aligned}
\sigma_{i j} & =2 W_{i j}-W_{i i}-W_{i j}>0, \quad i, j=1,2,3, \quad \text { and } i \neq j, \\
M_{1} & =W_{33}\left(W_{22}-W_{12}\right)+W_{23}\left(W_{12}-W_{23}\right)+W_{13}\left(W_{23}-W_{22}\right)>0, \\
M_{2} & =W_{11}\left(W_{33}-W_{23}\right)+W_{13}\left(W_{23}-W_{13}\right)+W_{12}\left(W_{13}-W_{33}\right)>0
\end{aligned}
$$

and

$$
M_{3}=W_{23}\left(W_{12}-W_{11}\right)+W_{12}\left(W_{13}-W_{12}\right)+W_{22}\left(W_{11}-W_{13}\right)>0
$$

in the notation of Edwards (1977).

For system (10),

$$
\begin{aligned}
& \sigma_{12}=2\left(1-h_{1}\right) \varepsilon_{1}, \quad \sigma_{13}=2\left(1-h_{2}\right) \delta_{2}, \quad \sigma_{23}=\sigma_{12}+\sigma_{13}, \\
& M_{1}=2 \varepsilon_{1} \varepsilon_{2}\left(1-h_{1}\right)+2 \delta_{1} \delta_{2}\left(1-h_{2}\right)+2 \varepsilon_{1} \delta_{2}\left(2 h_{1} h_{2}-h_{1}-h_{2}\right), \\
& M_{2}=2\left(\varepsilon_{1}-\delta_{1}\right) \delta_{2}\left(1-h_{2}\right),
\end{aligned}
$$

and

$$
M_{3}=2\left(\delta_{2}-\varepsilon_{2}\right) \varepsilon_{1}\left(1-h_{1}\right) \text {. }
$$

Evidently, when $h_{1}=1$ or $h_{2}=1$, condition (13) cannot be met. For $h_{1}=h_{2}=\frac{1}{2}$, condition (13) reduces to $2 \delta_{1}>\varepsilon_{1}>\delta_{1}$ and $2 \varepsilon_{2}>\delta_{2}>\varepsilon_{2}$. The condition

$$
M=M_{1}+M_{2}+M_{3}>0,
$$

is necessary, though not sufficient, for protected polymorphism. In this case,

$$
M=4 \varepsilon_{1} \delta_{2}\left(1-h_{1}\right)\left(1-h_{2}\right)
$$

and

$$
\frac{\partial M}{\partial h_{i}}=-4 \varepsilon_{1} \delta_{2}\left(1-h_{j}\right) \leqq 0 \quad \text { where } j \neq i,
$$

so that $M$ declines from a maximum of $\varepsilon_{1} \delta_{2}$ at $h_{1}=h_{2}=\frac{1}{2}$ to zero for $h_{1}=1$ and/or $h_{2}=1$. Since protected polymorphism also requires all $M_{i} \in(0, M)$, the larger $M$ can be, the more readily this condition can be met, all other things being equal. Therefore, the maintenance of genetic variation is again facilitated by greater dominance, some degree of dominance being strictly necessary. 
(iii) Polymorphism conditions for multiplicative components

With the genetic system specified by (11), the $\sigma_{i j}$ and $M_{i}$ have the following values.

$$
\begin{aligned}
\sigma_{12}= & f \varepsilon_{1}\left(2-h_{1}\right)+2 \varepsilon_{1} \delta_{1}\left(h_{1}-1\right)>0 \\
\sigma_{13}= & V \delta_{2}\left(2-h_{2}\right)+2 \varepsilon_{2} \delta_{2}\left(h_{2}-1\right)>0 \\
\sigma_{23}= & \sigma_{12}+\sigma_{13}+2\left(\varepsilon_{1} \delta_{2}+\varepsilon_{2} \delta_{1}\right)>0 \\
M_{1}= & f^{2} \varepsilon_{1} \varepsilon_{2}\left(2-h_{1}\right)+W \varepsilon_{1} \delta_{2}\left(h_{1} h_{2}-h_{1}-h_{2}\right)+V^{2} \delta_{1} \delta_{2}\left(2-h_{2}\right) \\
& +f\left\{\varepsilon_{1} \varepsilon_{2}\left[2 \delta_{1}\left(h_{1}-1\right)+\delta_{2}\left(2 h_{2}-2 h_{1} h_{2}+h_{1}\right)\right]-\left(\varepsilon_{1}-\varepsilon_{2}\right)\left(\varepsilon_{2} \delta_{1}+\varepsilon_{1} \delta_{2}\right)\right\} \\
& +V\left\{\delta_{1} \delta_{2}\left[2 \varepsilon_{2}\left(h_{2}-1\right)+\varepsilon_{1}\left(2 h_{1}-2 h_{1} h_{2}+h_{2}\right)\right]+\left(\delta_{1}-\delta_{2}\right)\left(\varepsilon_{2} \delta_{1}+\varepsilon_{1} \delta_{2}\right)\right\} \\
& +2 \varepsilon_{1} \varepsilon_{2} \delta_{1} \delta_{2}\left(2 h_{1} h_{2}-h_{1}-h_{2}\right)+\left(\varepsilon_{1}-\varepsilon_{2}\right)\left(\delta_{1}-\delta_{2}\right)\left(\varepsilon_{2} \delta_{1}+\varepsilon_{1} \delta_{2}\right), \\
M_{2}= & W \varepsilon_{1} \delta_{2}\left(2-h_{2}\right)+V^{2} \delta_{1} \delta_{2}\left(h_{2}-2\right) \\
& +f\left[\varepsilon_{1} \varepsilon_{2} \delta_{2}\left(2 h_{2}-3\right)-\varepsilon_{2}^{2} \delta_{1}\right]+V\left\{\delta_{1} \delta_{2}\left[\varepsilon_{2}\left(3-2 h_{2}\right)+\varepsilon_{1}\left(h_{2}-2\right)\right]+\varepsilon_{1} \delta_{2}^{2}\right\} \\
& +2 \varepsilon_{1} \varepsilon_{2} \delta_{1} \delta_{2}\left(1-h_{2}\right)-\varepsilon_{2} \delta_{2}\left(\varepsilon_{2} \delta_{1}+\varepsilon_{1} \delta_{2}\right),
\end{aligned}
$$

and

$$
\begin{aligned}
M_{3}= & f^{2} \varepsilon_{1} \varepsilon_{2}\left(h_{1}-2\right)+W \varepsilon_{1} \delta_{2}\left(2-h_{1}\right) \\
& +f\left\{\varepsilon_{1} \varepsilon_{2}\left[\delta_{1}\left(3-2 h_{1}\right)+\delta_{2}\left(h_{1}-2\right)\right]+\delta_{2} \varepsilon_{1}^{2}\right\}+V\left[\delta_{1} \delta_{2} \varepsilon_{1}\left(2 h_{1}-3\right)-\varepsilon_{2} \delta_{1}^{2}\right] \\
& -\varepsilon_{1} \delta_{1}\left(\varepsilon_{1} \delta_{2}+\varepsilon_{2} \delta_{1}\right)-2 \varepsilon_{1} \varepsilon_{2} \delta_{1} \delta_{2}\left(h_{1}-1\right) .
\end{aligned}
$$

Again, condition (14) is necessary but not sufficient for protected triallelic polymorphism. In this case, $M$ reduces to

$$
\begin{aligned}
M= & W \varepsilon_{1} \delta_{2}\left(2-h_{1}\right)\left(2-h_{2}\right) \\
& +2 \varepsilon_{1} \delta_{2}\left[f \varepsilon_{2}\left(h_{2}-1\right)\left(2-h_{1}\right)+V \delta_{1}\left(h_{1}-1\right)\left(2-h_{2}\right)\right] \\
& +4 \varepsilon_{1} \varepsilon_{2} \delta_{1} \delta_{2}\left(h_{1}-1\right)\left(h_{2}-1\right)-\left(\varepsilon_{2} \delta_{1}+\varepsilon_{1} \delta_{2}\right)^{2} .
\end{aligned}
$$

For small $\varepsilon_{i}$ and $\delta_{j}$, both $\partial M / \partial h_{k}$ are strictly less than zero. With full dominance, $h_{1}=h_{2}=1, M=\varepsilon_{1} \delta_{2} W-\left(\varepsilon_{2} \delta_{1}+\varepsilon_{1} \delta_{2}\right)^{2}$, which will be greater than zero for reasonable $\varepsilon_{i}$ and $\delta_{j}$ values. With strict additivity, $h_{1}=h_{2}=2$ and $M=-\left(\varepsilon_{2} \delta_{1}-\varepsilon_{1} \delta_{2}\right)^{2}<0$, precluding protected triallelic polymorphism. The degree of dominance plays essentially the same role as in the previous models.

\section{(iv) Genetic analysis}

The formula for the unique equilibrium gene frequency of $\boldsymbol{A}_{i}$, say $\hat{p}_{i}$, is $\hat{p}_{i}=M_{i} / M$, when conditions (12) are met (Edwards, 1977). These are too complex in this case to be written out usefully.

The algebra of genetic variance components is considerably more complex for three alleles at one locus compared with that for two. Rather than give overly cumbersome formulae, three numerical examples will be given for the first fitness component $\left(W_{1}\right)$ with $\varepsilon_{1}=\varepsilon_{2}=\varepsilon$. Thus, the array of 
phenotypes reduces to

$$
\begin{gathered}
W_{1}\left(A_{1} A_{1}\right)=W_{1}\left(A_{2} A_{3}\right)=V, \quad W_{1}\left(A_{1} A_{2}\right)=V+\varepsilon, \\
W_{1}\left(A_{2} A_{2}\right)=V+2 h_{1} \varepsilon, \quad W_{1}\left(A_{3} A_{3}\right)=V-2 \varepsilon, \\
\text { and } \quad W_{1}\left(A_{1} A_{3}\right)=V-\varepsilon, \quad \text { with } \quad 1 \geqq h_{1} \geqq \frac{1}{2} .
\end{gathered}
$$

Let the population mean be $\mu$ and the average effect of allele $j$ be $\alpha_{j}$, where $\alpha_{j}=\sum_{i} p_{i}\left(W_{1}\left(A_{i} A_{j}\right)-\mu\right)$. Then $V_{A}=2 \sum_{j} p_{j} \alpha_{j}^{2}$ and $V_{D}=$ $\sum_{i} \sum_{j} p_{i} p_{j}\left[W_{1}\left(A_{i} A_{j}\right)-\alpha_{i}-\alpha_{j}-\mu\right]^{2}$ (Kempthorne, 1957, p. 321).

$$
\begin{aligned}
\text { Case }(a): \quad p_{2}=0.2, \quad p_{1}=p_{3}=0.4 \\
\mu=V+\varepsilon\left(0.08 h_{1}-0.48\right), \quad \alpha_{1}=\varepsilon\left(0.28-0.08 h_{1}\right) \\
\alpha_{2}=\varepsilon\left(0.88+0.32 h_{1}\right), \quad \alpha_{3}=-\varepsilon\left(0.72+0.08 h_{1}\right), \\
V_{A}=0.4 \varepsilon^{2}\left[0.128 h_{1}^{2}+0.704 h_{1}+1.968\right] \\
V_{D}=0.120 \varepsilon^{2}\left(h_{1}-1\right)^{2} .
\end{aligned}
$$

With full dominance, $h_{1}=\frac{1}{2}, V_{A}=0.8704 \varepsilon^{2}$, and $V_{D}=0.03 \varepsilon^{2}$, so that $V_{D}$ is 3.45 per cent of $V_{A}$.

$$
\begin{gathered}
\text { Case }(b): \quad p_{1}=p_{2}=p_{3}=0.3 \dot{3} \\
\mu=V+0.2 \dot{2} \varepsilon\left(h_{1}-1\right), \quad \alpha_{1}=-0.2 \dot{2} \varepsilon\left(h_{1}-1\right) \\
\alpha_{2}=\varepsilon\left(0.5 \dot{5}+0.4 \dot{4} h_{1}\right), \quad \alpha_{3}=-\varepsilon\left(0.7 \dot{7}+0.2 \dot{2} h_{1}\right) \\
V_{A}=0.049 \varepsilon^{2}\left(4 h_{1}^{2}+10 h_{1}+15\right), \quad V_{D}=0.198 \varepsilon^{2}\left(h_{1}-1\right)^{2} .
\end{gathered}
$$

With full dominance, $V_{A}=1.029 \varepsilon^{2}$ and $V_{D}=0.0495$, so that $V_{D}$ is 4.81 per cent of $V_{A}$.

$$
\begin{gathered}
\text { Case }(c): \quad p_{2}=0.5, \quad p_{1}=p_{3}=0.25 \\
\mu=V+0.5 h_{1}, \quad \alpha_{1}=\varepsilon\left(0.25-0.5 h_{1}\right) \\
\alpha_{2}=\varepsilon\left(0.25+0.5 h_{1}\right), \quad \alpha_{3}=-\varepsilon\left(0.7+0.5 h_{1}\right) \\
V_{A}=\varepsilon^{2}\left(0.25 h_{1}^{2}+0.5 h_{1}+0.375\right), \quad V_{D}=0.25 \varepsilon^{2}\left(h_{1}-1\right)^{2} .
\end{gathered}
$$

With full dominance, $V_{A}=0.6875 \varepsilon^{2}$ and $V_{D}=0.0625 \varepsilon^{2}$, so that $V_{D}$ is 9.09 per cent of $V_{A}$.

As was the case for the diallelic models, even with full dominance $V_{D}$ is a small proportion of the total genetic variance at intermediate gene frequencies. Similarly, larger values of $p_{2}$ give proportionately greater $V_{D}$ values.

\section{TWO DIALLELIC LOCI}

(i) Genetic specification

Let there be two loci, $A$ and $B$, each with two alleles: $A$ and $a, B$ and $b$, respectively. Further, it is assumed that there is no epistatic interaction between loci in their effects on each fitness component separately. (Here additive non-epistatis is assumed.) With this assumption, the fitnesscomponent effects of each one-locus genotype can be specified independently, as follows. (All variables are positive and $0 \leqq h_{i j} \leqq 1$.) 


$\begin{array}{ccc} & W_{1} \text { Effect } & W_{2} \text { Effect } \\ A A & h_{11} \varepsilon_{1} & -\delta_{1} \\ A a & 0 & 0 \\ a a & -\varepsilon_{1} & h_{12} \delta_{1} \\ B B & h_{21} \varepsilon_{2} & -\delta_{2} \\ B b & 0 & 0 \\ b b & -\varepsilon_{2} & h_{22} \delta_{2}\end{array}$

These specifications are direct extensions of the one-locus system (1).

With additive fitness components, and no position effects, the nine distinct genotypes have the following fitness phenotypes, themselves lacking epistasis.

$$
\begin{aligned}
W_{11} & =W+h_{11} \varepsilon_{1}+h_{21} \varepsilon_{2}-\delta_{1}-\delta_{2} \\
W_{13} & =W+h_{21} \varepsilon_{2}-\delta_{2} \\
W_{33} & =W-\varepsilon_{1}+h_{21} \varepsilon_{2}+h_{12} \delta_{1}-\delta_{2} \\
W_{12} & =W+h_{11} \varepsilon_{1}-\delta_{1} \\
W_{14} & =W_{23}=W \\
W_{34} & =W-\varepsilon_{1}+h_{12} \delta_{1} \\
W_{22} & =W+h_{11} \varepsilon_{1}-\varepsilon_{2}-\delta_{1}+h_{22} \delta_{2} \\
W_{24} & =W-\varepsilon_{2}+h_{22} \delta_{2} \\
W_{44} & =W-\varepsilon_{1}-\varepsilon_{2}+h_{12} \delta_{1}+h_{22} \delta_{2} \\
W & =V+f,
\end{aligned}
$$

where $W_{i j}=W$ (gamete $i$ with gamete $j$ ), and $A B, A b, a B$, and $a b$ are gametes $1,2,3$ and 4 , respectively.

With multiplicative fitness components, and no position effects, the nine distinct genotypes have the following fitness phenotypes, which do exhibit epistasis, as specified by the $e_{i}$ parameters.

$$
\begin{aligned}
& W_{11}=W+a_{1}+a_{2}-e_{1} \\
& W_{13}=W+a_{2} \\
& W_{33}=W+a_{2}+a_{3}+e_{3} \\
& W_{12}=W+a_{1} \\
& W_{23}=W=W_{14} \\
& W_{34}=W+a_{3} \\
& W_{22}=W+a_{1}+a_{4}+e_{2} \\
& W_{24}=W+a_{4} \\
& W_{44}=W+a_{3}+a_{4}-e_{4}
\end{aligned}
$$




$$
\begin{aligned}
& a_{1}=f h_{11} \varepsilon_{1}-V \delta_{1}-h_{11} \varepsilon_{1} \delta_{1} \\
& a_{2}=f h_{21} \varepsilon_{2}-V \delta_{2}-h_{21} \varepsilon_{2} \delta_{2} \\
& a_{3}=V h_{12} \delta_{1}-f \varepsilon_{1}-h_{12} \varepsilon_{1} \delta_{1} \\
& a_{4}=V h_{22} \delta_{2}-f \varepsilon_{2}-h_{22} \varepsilon_{2} \delta_{2} \\
& e_{1}=h_{11} \varepsilon_{1} \delta_{2}+h_{21} \varepsilon_{2} \delta_{1} \\
& e_{2}=h_{11} h_{22} \varepsilon_{1} \delta_{2}+\varepsilon_{2} \delta_{1} \\
& e_{3}=\varepsilon_{1} \delta_{2}+h_{12} h_{21} \varepsilon_{2} \delta_{1} \\
& e_{4}=h_{22} \varepsilon_{1} \delta_{2}+h_{12} \varepsilon_{2} \delta_{1} \\
& W=V f .
\end{aligned}
$$

Not only does system (18) exhibit epistasis, it also does not, in general, have the form of the common "symmetric" two-locus system (cf. Karlin and Feldman, 1970).

\section{(ii) Polymorphism conditions for additive components}

With genetic system (17), the necessary and sufficient conditions for asymptotically stable polymorphism with all four alleles present are obtained directly from single locus conditions (Bodmer and Felsenstein, 1967). In the present notation, these conditions are:

$$
\begin{aligned}
& \delta_{1}>h_{11} \varepsilon_{1} \\
& \delta_{2}>h_{21} \varepsilon_{2} \\
& \varepsilon_{1}>h_{12} \delta_{1} \\
& \varepsilon_{2}>h_{22} \delta_{2} .
\end{aligned}
$$

As with the one-locus additive component models, polymorphism depends on sufficient dominance. With all $h_{i j}=1$, condition (19) cannot be met. With all $h_{i j}=0$, condition (19) is necessarily met for strictly positive $\delta_{k}$ and $\varepsilon_{l}$. Equilibrium gene frequencies for each locus may be found from the normal formulas for the one-diallelic-locus system. Furthermore the genetic variances are the sums of genetic variances for individual loci (Falconer, 1960, p. 138). In effect, nothing new arises from generalization of the one-locus model, in the absence of individual fitness-component epistasis.

(iii) Polymorphism conditions for multiplicative components

In the case of two-locus systems which are in general asymmetrically epistatic, there are no analytical tools ready-made for finding necessary and sufficient conditions for asymptotically stable polymorphism (Karlin, 1975). However, there are means by which sufficient, though not necessary, conditions for polymorphism may be found for two diallelic loci (cf. Bodmer and Felsenstein, 1967). This will be the avenue of analysis here.

With full dominance and nontrivial antagonistic pleiotropy, the twolocus system specified by (18) always exhibits protected polymorphism, as 
will be ascertained. The analysis of protected polymorphism conditions, though not local equilibrium dynamics, can be readily understood in terms of the representation of fig. 2. The four corners of fig. 2, at which both loci are homozygotic, are unstable if the marginal fitness of the heterozygote for either locus is greater than that of the corner homozygote (Bodmer and Felsenstein, 1967). In this case, it is trivial to show that corner instability necessarily arises with full dominance when $V>\sup \left(\varepsilon_{1}, \varepsilon_{2}, \delta_{1}, \delta_{2}\right)$ and $f>\sup \left(\varepsilon_{1}, \varepsilon_{2}, \delta_{1}, \delta_{2}\right)$, as has been assumed. Therefore, polymorphism depends on the instability of any one-locus equilibria on the four edges of fig. 2.

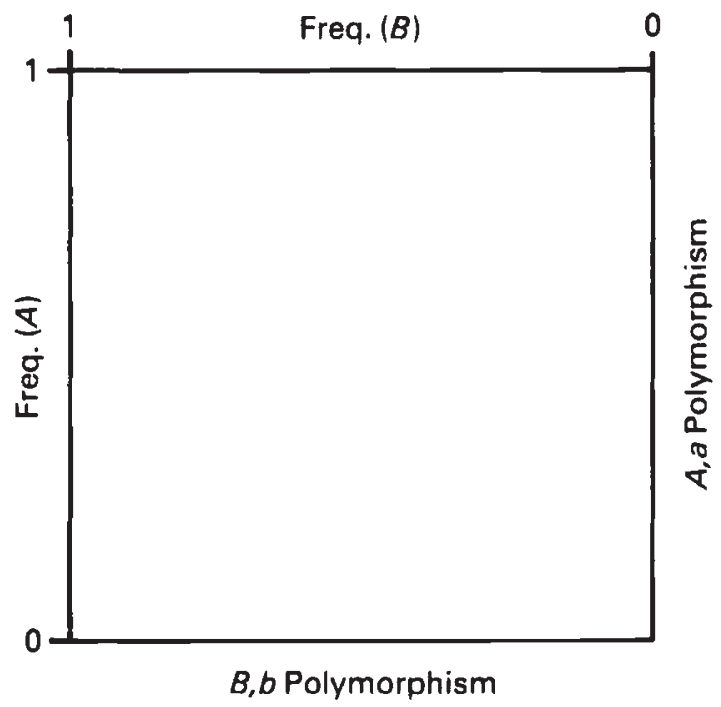

FIG. 2.-A partial representation of the evolutionary state-space of a two diallelic loci system, with the vertical dimension representing the frequency of allele $A$ and the horizontal dimension representing the frequency of allele $B$.

For the bottom edge, for example, Bodmer and Felsenstein (1967) show that instability with an arbitrary non-zero recombination frequency requires:

$$
\begin{array}{ll}
\left(\mathrm{W}-W_{2}^{*}\right) v \leqq\left(W_{1}^{*}-\mathrm{W}\right) u, & \text { when } W_{1}^{*}>W_{2}^{*}, \\
\left(\mathrm{~W}-W_{1}^{*}\right) u \leqq\left(W_{2}^{*}-\mathrm{W}\right) v, & \text { when } W_{2}^{*}>W_{1}^{*},
\end{array}
$$

or

$$
W_{1}^{*} \geqq \mathrm{~W}, \text { when } W_{1}^{*}=W_{2}^{*} \text {, }
$$

where

$$
\begin{gathered}
u=\left(W_{34}-W_{44}\right) /\left(2 W_{34}-W_{33}-W_{44}\right), \quad v=1-u, \\
W_{1}^{*}=u W_{13}+v W_{14}, \quad W_{2}^{*}=u W_{14}+v W_{24}, \quad \text { and } \quad W=u W_{33}+v W_{34} .
\end{gathered}
$$

Analogous conditions determine the instability of the other three edges, with appropriate changes in $u, v, W_{1}^{*}, W_{2}^{*}$, and $\mathrm{W}$. 
For the four edges of fig. 2, after some algebra these conditions reduce to:

$$
\begin{aligned}
& \left(V \delta_{1}+f \varepsilon_{1}-\varepsilon_{1} \delta_{2}\right)^{2} \geqq V \varepsilon_{1} \delta_{1}^{2} \\
& \left(V \delta_{2}+f \varepsilon_{2}-\varepsilon_{2} \delta_{1}\right)^{2} \geqq V \varepsilon_{2} \delta_{2}^{2} \\
& \left(V \delta_{1}+f \varepsilon_{1}-\varepsilon_{2} \delta_{1}\right)^{2} \geqq f \delta_{1} \varepsilon_{1}^{2} \\
& \left(V \delta_{2}+f \varepsilon_{2}-\varepsilon_{1} \delta_{2}\right)^{2} \geqq f \delta_{2} \varepsilon_{2}^{2} .
\end{aligned}
$$

In the case of (21a), for example, since $f>\delta_{2}$, condition (21a) is met if $\left(V \delta_{1}\right)^{2} \geqq V \varepsilon_{1} \delta_{1}^{2}$, and thus if $V \geqq \varepsilon_{1}$, which has been assumed. Likewise, the other three conditions are met for $V, f>\delta_{i}, \varepsilon_{j}>0$. Thus full dominance guarantees protected polymorphism, as before.

This analysis may be generalized to treat the boundaries of the general two-locus system specified by (18). As was the case for the full-dominance system, asymptotic polymorphism is necessarily achieved if all corners and all non-transient edges are unstable. The sufficient conditions for corner instability are $e_{1}>a_{2}$ or $e_{1}>a_{1}, 0>e_{2}+a_{1}$ or $0>e_{2}+a_{4}, 0>e_{3}+a_{3}$ or $0>e_{3}+a_{2}$, and $e_{4}>a_{3}$ or $e_{4}>a_{4}$. These conditions are always met when each $a_{i}$ is less than $-E$, where $E=\varepsilon_{1} \delta_{2}+\varepsilon_{2} \delta_{1}$, which in turn requires

$$
\begin{aligned}
& V \delta_{1}>h_{11}\left(f-\delta_{1}\right) \varepsilon_{1}+E \\
& V \delta_{2}>h_{21}\left(f-\delta_{2}\right) \varepsilon_{2}+E \\
& f \varepsilon_{1}>h_{12}\left(V-\varepsilon_{1}\right) \delta_{1}+E \\
& f \varepsilon_{2}>h_{22}\left(V-\varepsilon_{2}\right) \delta_{2}+E .
\end{aligned}
$$

This condition can be met by $h_{i j}$ sufficiently small providing the $\varepsilon_{i}$ and $\delta_{k}$ are all of similar magnitude and much less than both $V$ and $f$.

With all corners unstable, only the instability of all the edges remains as a sufficient condition for asymptotic polymorphism. Using the same method of analysis as that used to find condition (21) in the complete dominance case, the sufficient condition for instability for the bottom edge is

$$
W_{1}^{*}-\mathrm{W}=-a_{3}+\left(\frac{a_{4}-e_{4}}{-a_{2}-a_{4}-e_{3}+e_{4}}\right) e_{3}>0
$$

providing

$$
\begin{aligned}
W_{2}^{*}-W_{1}^{*}= & \frac{e_{3} a_{4}+a_{2} a_{4}}{a_{2}+a_{4}+e_{3}-e_{4}} \\
= & f \varepsilon_{2} \varepsilon_{1} \delta_{2}\left(1-h_{22} h_{21}\right)+V \delta_{2} \varepsilon_{2} \delta_{1}\left(1-h_{21} h_{22}\right) \\
& \frac{+\varepsilon_{2} \delta_{2}\left[\varepsilon_{2} \delta_{2} h_{22}\left(1+h_{21}\right)+\varepsilon_{2} \delta_{1} h_{12} h_{21}\left(1+h_{22}\right)\right]}{f \varepsilon_{2}\left(1-h_{21}\right)+\left(V-\varepsilon_{1}\right) \delta_{2}\left(1-h_{22}\right)} \\
& \quad+\varepsilon_{2} \delta_{1} h_{12}\left(1-h_{21}\right)+\varepsilon_{2} \delta_{2}\left(h_{21}+h_{22}\right) \\
& >0,
\end{aligned}
$$

which will always hold when $V>\varepsilon_{1}$, as is assumed here. Therefore, edge instability is achieved if

$$
-a_{3}>e_{3}\left(\frac{a_{4}-e_{4}}{a_{2}+a_{4}+e_{3}-e_{4}}\right)
$$


or, since from (20) and (22)

$$
-\left(a_{2}+a_{4}+e_{3}-e_{4}\right) \geqq e_{4}-a_{4},
$$

or, finally, if

$$
f \varepsilon_{1} \geqq h_{12}\left(V-\varepsilon_{1}\right) \delta_{1}+E,
$$

as specified by $(22 \mathrm{c})$. Similarly, all other sufficient edge instability conditions reduce to the sufficient corner instability conditions (22a), (22b), and (22d). Thus the complete condition (22) is sufficient for protected polymorphism for genes of small effects. Except for the factor $E$, these conditions are much like condition (3) for the single-locus case. And since $E$ is quite small under the conditions assumed here, these sufficient conditions for two-locus polymorphism have essentially the same dependence on dominance as those for the multiplicative components one-locus model.

However, dominance is not a necessary condition for stable polymorphism in the multiplicative case. Some special cases of the multiplicative-component, additive-inheritance, two-locus system are symmetric, and particular examples of local, asymptotically stable, polymorphic equilibrium with sufficiently low recombination can be found using the formulas of Karlin and Feldman (1970). This difference between multiplicative and additive components with two diallelic loci is analogous to that with one diallelic locus.

\section{Discussion}

It has been shown for a group of simple models that protected polymorphism is readily guaranteed when there is antagonistic pleiotropy between additive or multiplicative fitness components. In addition, it has been shown that some degree of directional dominance, tending to produce recessive deleterious gene action, fosters the establishment of such protected polymorphism. Nevertheless, this dominance in gene action need not lead to large dominance genetic variances relative to additive genetic, or genic, variance.

Three important points remain for discussion. (1) Are the results found for these models robust, or are they model-dependent? (2) What is the bearing of available experimental results on these models? (3) What further experiments could be done to test these models, or their assumptions?

(1) The models considered here are based on the most convenient assumptions for analysis, and many further complications deserve examination. A few examples should suffice. (a) Dioecy. With two sexes, the conditions on the preservation of genetic polymorphism in general become less stringent (cf. Ewens, 1979, p. 39). Evidently, with two sexes and several fitness-components, while the algebra may prove impossible, it should be easier, both numerically and biologically, for antagonistic pleiotropy to lead to genetic polymorphism. Whether or not the results concerning dominance which have been found here will hold is another matter. An increase in the extent of dominance of the favourable allele over the deleterious allele fosters polymorphism in some single-component dioecious models (Kidwell et al., 1977). This suggests that conflating the two models together might allow still greater scope for the action of dominance in determining the outcome of selection. (b) More than three alleles at one 
locus. Though many of the features of diallelic one-locus theory hold for an arbitrary number of alleles at a single locus, the role of the magnitude of dominance with antagonistic pleiotropy seems difficult to generalize. Even in the triallelic model analysed here, a special pattern of dominance was used to simplify the resulting equations. More general formulations may be completely uninterpretable. (c) Overlapping generations and agestructure. Unfortunately, with even the simplest overlapping generations models, fitness depends on the Malthusian parameter, which has a nonlinear dependence on viability and fecundity characters through the Euler-Lotka characteristic equation (vid. Charlesworth, 1980). Since the models developed here depend on simple addition or multiplication of fitness-components, they are no more than suggestive of implications for the quantitative genetics of fitness components with overlapping generations.

Thus, while the simple analyses given here are of general significance in showing that antagonistic pleiotropy could, in principle, be important in the maintenance of genetic variation, it seems very difficult to infer what role dominance plays in more realistic genetic systems.

(2) Until recently, it was widely accepted that there was relatively little additive genetic variation for fitness-components. This was inferred from the widespread pattern of low heritabilities among characters such as conception rate in cattle, litter size in pigs, viability in poultry, and egg production in Drosophila (cf. Falconer, 1960, pp. 167-168). This pattern was taken to reflect the general validity of Fisher's Fundamental Theorem, with its corollary that natural selection should act to use up additive genetic variability for fitness (Istock, 1981).

Unfortunately, most of these heritability studies were performed either on domesticated animals which had been subjected to artificial selection for these characters and/or inbreeding, or on small laboratory populations descended from extremely small numbers of wild-caught founders (cf. Robertson, 1957). Subsequent studies of laboratory populations which have been sampled from nature and maintained so as to preserve their genetic variability have revealed an entirely different pattern. Dingle et al. (1977), Istock (1981), and Rose and Charlesworth (1980) all provide evidence of substantial additive genetic variation for characters which are components of fitness. Moreover, there is now evidence for significant antagonistic pleiotropy between fitness-components, especially in Drosophila (Rose and Charlesworth, 1980; Simmons et al., 1980). Evidently, these results are in conformity with the assumptions and predictions of the models presented here, though no experimental "proofs" are available for these models.

(3) If antagonistic pleiotropy acts to maintain the abundant additive genetic variation for fitness-components which has been discovered recently, then it should be possible to find clear evidence for this by means of relatively simple experiments. With the large additive genetic variances for individual fitness-components which are predicted come heritabilities sufficiently large to make artificial selection on these characters practical. Thus it should be possible to select for a substantial increase in individual fitness-components. If this can indeed be done, there should be a decrease in those other fitness-components which are subject to antagonistic pleiotropy with the selected fitness-component(s). This has in fact been 
observed (e.g., Rose and Charlesworth, 1980). However, this pattern of antagonistic response could also be due to inbreeding, mutation accumulation, linkage disequilibrium between selected alleles and deleterious alleles at different loci, or a combination of these factors. Still, these alternative explanations could be evaluated experimentally using crosses of selected lines, selection relaxation, reversed selection, or some combination of these methods.

In all likelihood, the specific patterns of gene action, and thus the validity of the specific models, studied here may be impossible to analyse experimentally for purely practical reasons. Nonetheless, the possibilities for interaction between theory and experiment must be judged relatively promising, compared with the prevailing difficulty of bringing them together in population genetics as a whole.

Acknowledgments. - Research conducted while the author was a N.A.T.O. postdoctoral fellow. J. F. Crow, M. E. Moody, and P. A. Otto provided helpful comments on the manuscript.

\section{REFERENCES}

BODMER, W. F., AND FELSENSTEIN, J. 1967. Linkage and selection: Theoretical analysis of the deterministic two locus random mating model. Genetics, 57, 237-265.

CASPARI, E. 1950. On the selective value of the alleles $R t$ and $r t$ in Ephestia kühniella. Am. Nat., 84, 367-380.

Charlesworth, B. 1980. Evolution in Age-Structured Populations. Cambridge University Press, Cambridge, U.K.

DINGLE, H., BROWN, C. K., AND HEgMANN, J. P. 1977. The nature of genetic variance influencing photoperiodic diapause in a migrant insect, Oncopeltus fasciatus. Amer. Nat., $111,1047-1059$.

EDWARDS. A. W. F. 1977. Foundations of Mathematical Genetics. Cambridge University Press, Cambridge, U.K.

EWENS, W. J. 1979. Mathematical Population Genetics. Springer-Verlag, New York.

FALCONER, D. S. 1960. Introduction to Quantitative Genetics. Oliver and Boyd, Edinburgh.

FALCONER, D. S. 1977. Why are mice the size they are? In International Conference on Quantitative Genetics, eds. E. Pollak, O. Kempthorne, and E. J. Bailey. Iowa State University Press, Ames lowa.

FISHER, R. A. 1918. The correlation between relatives on the supposition of Mendelian inheritance. Proc. Roy. Soc. Edin., 52, 399-433.

ISTOCK, C. A. 1981. The extent and consequences of heritable variation for fitness characters. In Population Biology: Retrospect and Prospect, eds. C. R. King and P. S. Dawson. Columbia University Press, New York.

KARLIN, S. 1975. General two-locus selection models: Some objectives, results and interpretations. Theor. Pop. Biol. 7, 364-398.

KARLIN, S., AND FELDMAN, M. W. 1970. Linkage and selection: Two locus symmetric viability model. Theor. Pop. Biol., 1, 39-71.

KEMPTHORNE, O. 1957. An Introduction to Genetic Statistics. Wiley, New York.

KIDWELL, J. F., CLEGG, M. T., STEWART, F. M., AND PROUT, T. 1977. Regions of stable equilibria for models of differential selection in the two sexes under random mating. Genetics, 85, 171-183.

LANDE, R. 1980. The genetic covariance between characters maintained by pleiotropic mutations. Genetics, 94, 203-215.

MUKAI, T. 1977. Lack of experimental evidence supporting selection for the maintenance of isozyme polymorphisms in Drosophila melanogaster. Proc. Taniguchi Int. Symp. Biophys., $2,103-126$.

ROBERTSON, F. W. 1957. Studies in quantitative inheritance. XI. Genetic and environmental correlation between body size and egg production in Drosophila melanogaster. J. Genet., $55,428-443$. 
ROSE, M., AND CHARLESWORTH, B. 1980. A test of evolutionary theories of senescence. Nature, 287, 141-142.

SIMMONS, M. J., PRESTON, C. R., AND ENGELS, W. R. 1980. Pleiotropic effects on fitness of mutations affecting viability in Drosophila melanogaster. Genetics, 94, 467-475.

WAllaCE, B. 1959. The role of heterozygosity in Drosophila populations. Proc. X. Int. Congr. Genetics. 1, 408-419.

WAllace, B. 1968. Topics in Population Genetics. Norton \& Co., New York.

WAllaCE, B. 1970. Genetic Load. Prentice-Hall, Englewood Cliffs, N.J.

WRIGHT, S. 1968. Evolution and the Genetics of Populations. I. Genetic and Biometric Foundations. University of Chicago Press, Chicago.

WRIGHT, s. 1977. Evolution and the Genetics of Populations. III. Experimental Results and Evolutionary Deductions. University of Chicago Press, Chicago. 